Research Paper

\title{
Regulation of Androgen Receptor Expression Alters AMPK Phosphorylation in the Endometrium: In Vivo and In Vitro Studies in Women with Polycystic Ovary Syn- drome
}

Xin $\mathrm{Li}^{1,2,3^{*}}$, Bano Pishdari ${ }^{*}$, Peng Cui ${ }^{*}$, Min Hu${ }^{1}$, Hong-Ping Yang 2,3 , Yan-Rong Guo ${ }^{2,3}$, Hong-Yuan Jiang 2,3 , Yi Feng ${ }^{1,4}$, Håkan Billig1 and Ruijin Shao ${ }^{1 凶}$

1. Department of Physiology/Endocrinology, Institute of Neuroscience and Physiology, The Sahlgrenska Academy, University of Gothenburg, Gothenburg, Sweden.

2. Department of Gynecology, Obstetrics and Gynecology Hospital of Fudan University, Shanghai, China;

3. Shanghai Key Laboratory of Female Reproductive Endocrine Related Diseases, Shanghai, China;

4. Department of Integrative Medicine and Neurobiology, State Key Lab of Medical Neurobiology, Shanghai Medical College and Institute of Acupuncture Research (WHO Collaborating Center for Traditional Medicine), Institute of Brain Science, Fudan University, Shanghai, China.

* These authors contributed equally to the study.

$\bowtie$ Corresponding author: Ruijin Shao, M.D., Ph.D., Tel: +46 31 7863408; Fax: +46 31 7863512; E-mail: ruijin.shao@fysiologi.gu.se Home page: http://www.neurophys.gu.se/sektioner/fysiologi/endo/staff/ruijin_shao/

() 2015 Ivyspring International Publisher. Reproduction is permitted for personal, noncommercial use, provided that the article is in whole, unmodified, and properly cited. See http://ivyspring.com/terms for terms and conditions.

Received: 2015.07.01; Accepted: 2015.10.12; Published: 2015.11.01

\begin{abstract}
The failure of reproductive success in polycystic ovary syndrome (PCOS) patients could be in part due to endometrial dysfunction. However, no studies have investigated any causality between androgen, androgen receptor (AR) expression, and adenosine monophosphate activated protein kinase (AMPK) activation in the endometrium under physiological and pathological conditions. In the present study, we show that 1 ) endometrial AR expression levels fluctuate in non-PCOS and PCOS patients during the menstrual cycle; 2) the menstrual phase-dependent alteration of P-AMPKa expression occurs in non-PCOS patients but not in PCOS patients; 3) AR expression is higher in PCOS patients than non-PCOS patients during hyperplasia while AMPKa activation (indicated by the ratio of p-AMPKa to AMPKa); and 4) co-localization of AR and Ki-67 in epithelial cell nuclei is observed in endometrial hyperplasia. Importantly, using in vitro human tissue culture and an in vivo 5a-dihydrotestosterone-treated rat model, we show that the action of androgen on AMPKa activation is likely mediated through nuclear AR, especially in epithelial cells. Collectively, we present evidence that AR expression and AMPK $\alpha$ activation depend on menstrual cycle phase and the presence of PCOS, and the data suggest that AR-mediated regulation of AMPKa activation might play a role in the development of endometrial hyperplasia.
\end{abstract}

Key words: 5a-dihydrotestosterone, androgen receptor, AMPKa, endometrium, PCOS.

\section{Introduction}

Polycystic ovary syndrome (PCOS) is a complex, heterogeneous hormone-imbalance disorder [1] that occurs in approximately $4 \%-18 \%$ of adolescent and reproductive-aged women worldwide [2]. In addition to its negative effects on endocrine activity and metabolism, PCOS has adverse impacts on female re- production [1, 3]. For example, women with PCOS generally have chronic anovulation, and their endometria tend to remain in a proliferative state due to the lack of counterbalance by progesterone [4]. These defects result in about $75 \%$ of PCOS patients suffering from anovulation infertility [5], and about $50 \%$ of 
PCOS patients experience recurrent pregnancy loss [6]. It is generally believed that the failure of reproductive success in PCOS patients could be in part due to endometrial dysfunction $[7,8]$.

The human endometrium plays an essential role in menstruation, implantation, pregnancy, and endometrial repair [7] through alterations of epithelial and stromal cell proliferation, differentiation, secretion, and apoptosis [9]. The modulation of different protein expression and signaling pathways has been shown to influence endometrial function $[7,10]$. In addition to estrogen and progesterone [10], androgen is also essential for female reproduction through the activation of androgen receptor (AR), which belongs to a member of the steroid receptor superfamily and regulates transcription of target genes [11]. AR undergoes cyclical changes in expression and has different endometrial cell localization and regulation patterns in women during the menstrual cycle [10, 11]. For example, AR expression in human endometrium is predominantly localized to the stromal cells with maximal expression detected during the proliferative phase of the menstrual cycle [12-16]. Furthermore, studies undertaken in our laboratory and others have demonstrated that endometrial AR mRNA and protein expression are elevated in PCOS patients compared to non-PCOS patients [12, 17-21]. Because hyperandrogenism is one of the main clinical complications of PCOS [1, 3], accumulating evidence suggests that flutamide (an AR antagonist) therapy exerts beneficial ovarian, endocrine, and metabolic effects in PCOS patients [22-27]. However, an important unanswered question is how androgenic stimulation is involved in the endometrial function in PCOS patients under different phases of menstrual cycle and hyperplasia [28, 29]. Specifically, an understanding of the molecular mechanisms and/or consequences of increased AR expression in the endometrium in women with PCOS remains elusive.

Mammalian adenosine monophosphate-activated protein kinase (AMPK) is a multisubstrate, heterotrimeric serine/threonine kinase that consists of three subunits ( $\alpha, \beta$, and $\gamma)$ [30]. All three subunits function in unison to modulate cellular metabolism and to control cell growth [31, 32]. The a-subunit in particular has been shown to harbor AMPK activity because the activation of AMPK requires the phosphorylation of threonine 172 , which is located within the a catalytic subunit [32]. Although AMPK is expressed in all tissues, accumulating evidence indicates that there is considerable variation in tissue-specific expression, association, and subcellular localization of the individual subunits [33]. However, only AMPKa has been demonstrated in the endometria of women with PCOS and endometrial cancer [34,
35]. Furthermore, while hormonal, nutrient, and metabolic stress signals regulate AMPK activation and its activity in multiple peripheral tissues such as liver, skeletal muscle, and adipose tissues $[32,36]$ as well as different cancer cells [37], to our knowledge no studies have investigated any causality between androgen, AR expression, and AMPK activation in the endometrium under physiological and pathological conditions.

The aims of the present study, therefore, were to investigate the temporal and spatial expression patterns of AR, p-AMPKa, AMPKa, and Ki67 (a cellular marker for proliferation) in the endometrium of PCOS patients in different phases of the menstrual cycle or with hyperplasia. In addition, the effect of 5a-dihydrotestosterone (DHT) and flutamide on the regulation of $\mathrm{AR}$, $\mathrm{p}$-AMPKa, and AMPKa expression was investigated with in vitro human tissue culture and an in vivo rat model.

\section{Materials and methods}

\section{Reagents and antibodies}

DHT (a specific AR agonist, D-5027), flutamide (a specific AR antagonist, F-0397), and diaminobenzidine (DAB) were from Sigma-Aldrich (St. Louis, MO). Avidin-biotinylated-peroxidase complex detection system (ABC kit) was from Vector Laboratories Inc. (Burlingame, CA). All antibodies were from the following sources: AR (\#5153, this antibody reacts with human tissues), AMPKa (\#2532), phospho-AMPKa (phosphorylated threonine 172, \#2535), and Ki-67 (\#11882) antibodies were from Cell Signaling Technology (Danvers, MA); AR (sc-816, this antibody reacts with rodent tissues) and p21 ${ }^{\mathrm{Cip} 1}$ antibodies were from Santa Cruz Biotechnology (Santa Cruz, CA); and cysteine-rich protein 61 (CYR61, HPA029853) antibody was from Sigma-Aldrich. Anti-mouse IgG horseradish peroxidase (HRP)-conjugated goat (A2304) and anti-rabbit IgG HRP-conjugated goat (A0545) secondary antibodies were from Sigma-Aldrich. Alexa Fluor 594-conjugated goat polyclonal anti-mouse IgG, and Alexa Fluor 488-conjugated goat polyclonal anti-rabbit IgG were from Invitrogen (Life Technologies Europe BV, Stockholm, Sweden).

\section{Patients and tissue collection}

This investigation was approved by the institutional ethical review board of the Obstetrics and Gynecology Hospital of Fudan University, Shanghai, China, and signed informed consent for use of the samples was obtained from all patients. All endometrial tissues were obtained by endometrial curettage of the bisected uteri obtained during hysterectomy for benign gynecological indications. Each endometrial 
sample was diagnosed and staged by routine pathology analysis based on standard histological criteria [38], and the patient's last reported menstrual period was recorded at the time of collection [39]. Immunohistochemical staining of cysteine-rich 61 (Cyr61) with a confirmed histological diagnosis of simple or complex endometrial hyperplasia without cytological atypia was performed (Fig. 1). All fertile women ( $\mathrm{n}=$ 45 , aged $25-51$ years) at the proliferative $(n=19)$ or secretory phases of the menstrual cycle $(n=15)$ or with hyperplasia $(n=11)$ taking part in the investigation had regular menstrual cycles and showed no evidence of any pathological uterine disorder. Endometrial biopsies at different stages of the menstrual cycle were obtained from PCOS patients $(n=14$, aged 28-36 years) at the proliferative $(n=9)$ and secretory phases of the menstrual cycle $(n=2)$ or with hyperplasia $(n=$ 3). PCOS was diagnosed based on the Rotterdam cri-

H\&E
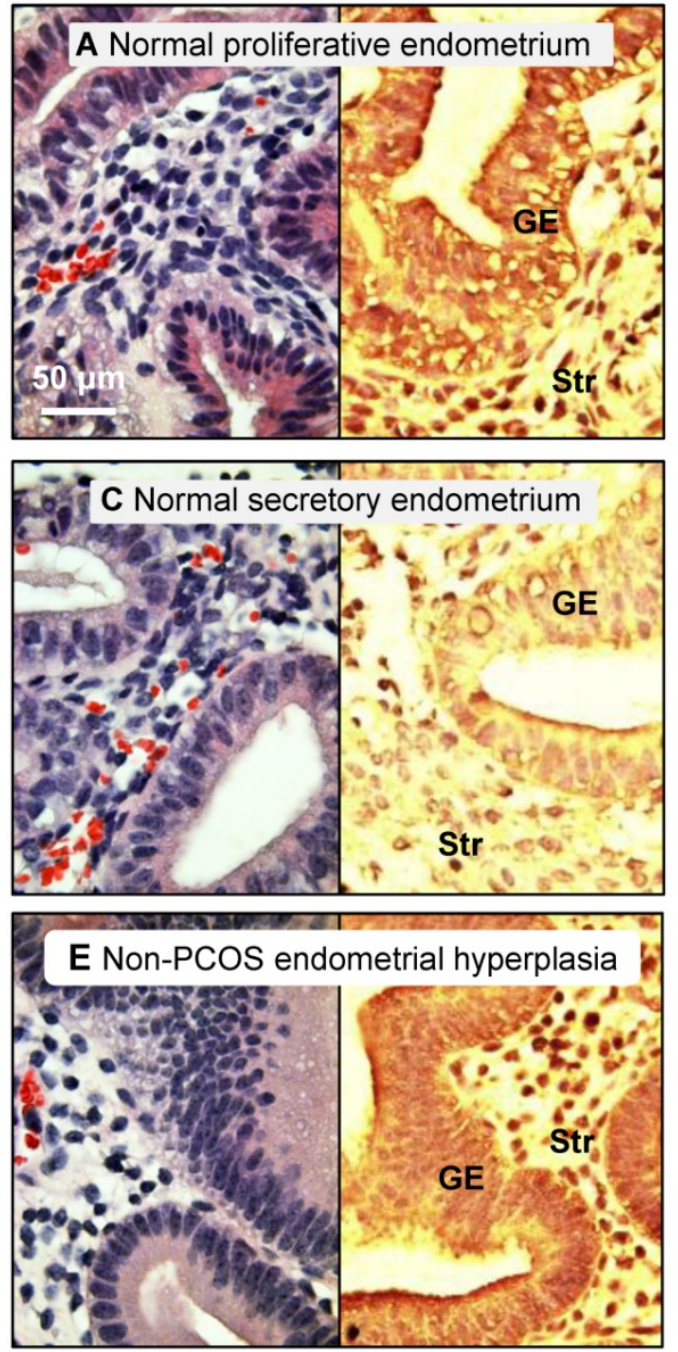

teria provided by the American Society for Reproductive Medicine and the European Society for Human Reproduction and Embryology [40]. All PCOS patients were diagnosed according to at least two of the following criteria: 1) oligo/anovulation, 2) signs of hyperandrogenism (i.e., hirsutism and acne) and/or biochemical measurements, or 3) enhanced ovaries (at least 12 discrete follicles of $2-9 \mathrm{~mm}$ in diameter in one ovary or an ovarian volume $>10 \mathrm{~cm}^{3}$ observed by transvaginal ultrasonography). No PCOS patients presented congenital adrenal hyperplasia, Cushing's syndrome, thyroid hormone abnormalities, hyperprolactinemia, or ovarian/adrenal tumors. None had any history of previous first-trimester miscarriage or pregnancy. Neither non-PCOS nor PCOS subjects were exposed to hormonal medication for at least three months prior to tissues sampling [8].
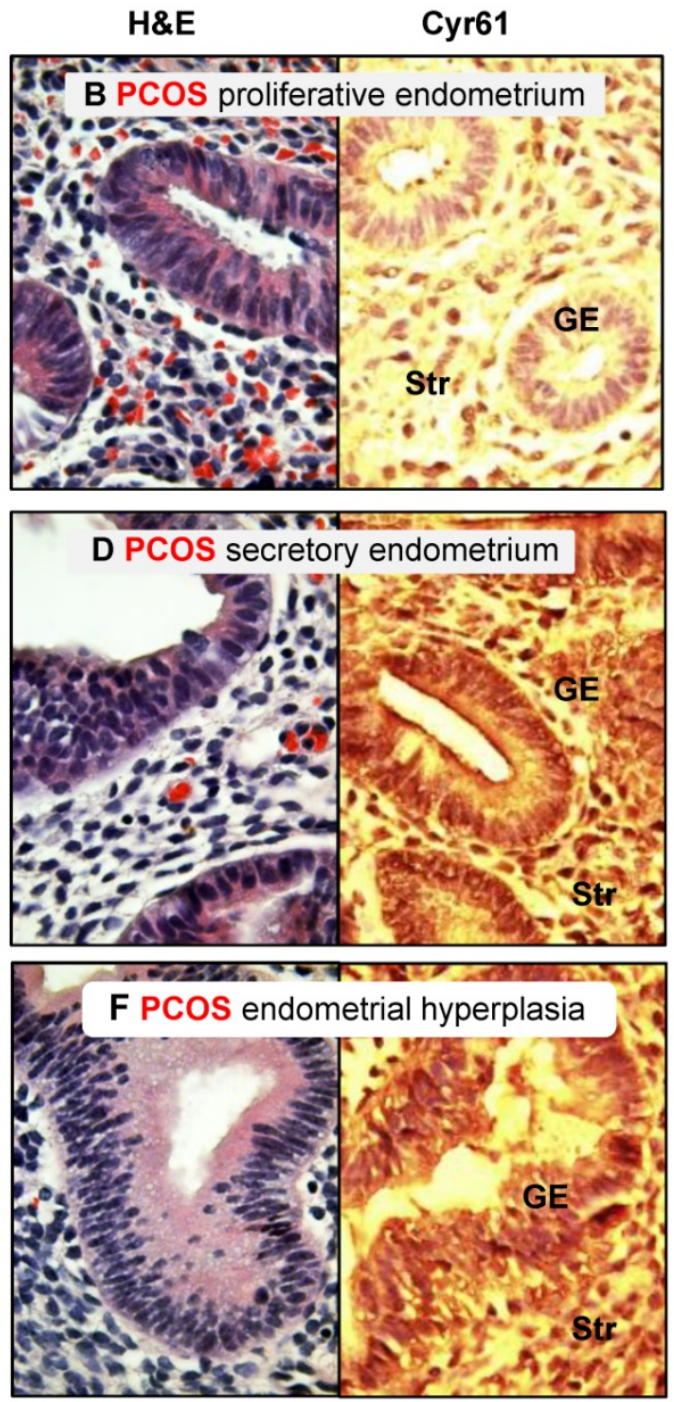

Figure 1. Distribution of cysteine-rich 61 (Cyr61) proteins in the endometrium in women with and without PCOS. Human endometrial tissues were fixed in formalin and embedded in paraffin, and immunohistochemistry was performed. It was reported that the expression of Cyr61, a cysteine-rich secreted heparin-binding protein, was significantly increased in the endometrium of PCOS patients with hyperplasia compared to age-matched, non-PCOS patients with normal menstrual cycles, and it appears to be a useful tissue biomarker for the detection of endometrial hyperplasia. Therefore, we used Cyr61 protein expression to verify that the endometrial samples had come from non-PCOS and PCOS patients. Representative images from three independent experiments are shown. The histology of hematoxylin/eosin-stained human endometrial biopsy samples is indicated. GE, glandular epithelial cells; Str, stromal cells. 


\section{Primary in vitro tissue culture}

Tissue culture was essentially carried out as described previously [21, 39]. Briefly, endometrial tissues from each woman in the proliferative phase of a normal menstrual cycle were treated with DHT (100 $\mathrm{nM})$ and were incubated in a humidified incubator $\left(37^{\circ} \mathrm{C}, 95 \% \mathrm{O}_{2}, 5 \% \mathrm{CO}_{2}\right)$ for $24 \mathrm{~h}$. On the second day, the tissues were further treated with DHT $(100 \mathrm{nM})$ or DHT $(100 \mathrm{nM})$ plus flutamide $(10 \mu \mathrm{M})$ and were cultured for an additional $24 \mathrm{~h}$. The selected doses of DHT and flutamide were according to previous studies [41, 42]. DHT and flutamide were dissolved in $100 \%$ ethanol at a concentration of $50 \mathrm{mM}$ and added to culture medium to achieve the desired final concentration. Controls were treated with $100 \%$ ethanol. At the end of the experiments, tissues were washed with ice cold PBS and were snap-frozen in liquid nitrogen and stored at $-70^{\circ} \mathrm{C}$.

\section{Hormonal treatment in animals}

This study was approved by the Animal Ethics Committee of the University of Gothenburg, Sweden. Prepubescent female Wistar rats obtained from Charles River (Sulzfeld, Germany) were used to avoid both the effects of endogenous estrogen and the surgical procedures such as ovariectomy that would be necessary to remove the main source of endogenous steroid hormone production. Rats $(\mathrm{n}=18)$ were randomly treated with vehicle only (control) or with DHT (Sigma-Aldrich, St. Louis, MO). There were nine animals in each experimental group. At 21 days of age, rats were treated with daily subcutaneous injections of $100 \mu \mathrm{l}$ sesame oil vehicle or DHT $(1.66 \mathrm{mg} / \mathrm{kg}$ in $100 \mu$ l sesame oil) for one week [43]. The dose and time of DHT injection has previously been shown to suppress hypothalamic AR expression in both female and male rats $[43,44]$. Animals were housed in a room under defined conditions (e.g., $21-22^{\circ} \mathrm{C}, 55-65 \%$ humidity and a 12 hour light/dark cycle). Rats had free access to tap water and were fed ad libitum with standard rodent chow. All animals were allowed to acclimate to the animal facilities for at least five days before initiation of the treatment and experiment. Under anesthesia, the uterine tissues were collected and fixed in $4 \%$ formaldehyde neutral buffered solution for $24 \mathrm{~h}$ at $4^{\circ} \mathrm{C}$ and embedded in paraffin for histochemical analysis.

\section{Western blot analysis}

Endometrial and uterine tissues were extracted according to the previous studies [21, 39]. A detailed explanation of the Western blot analysis and membrane stripping protocol has been published elsewhere [45]. Equal amounts of protein for each treatment group were resolved on NuPAGE 4-12\%
Bis-Tris gels (Invitrogen) and transferred onto PVDF membranes. The primary antibodies (1:1000-2000 dilution) were used. The specificity of AR antibodies (\#5153 and sc-816) is shown in Figure 2.

\section{Immunohistochemistry}

The tissues were fixed in $4 \%$ formaldehyde neutral buffered solution for $24 \mathrm{~h}$ at $4^{\circ} \mathrm{C}$. Following paraffin embedding and sectioning $(5 \mu \mathrm{m})$, sections were stained with hematoxylin and eosin (H\&E). Two different methods of immunohistochemistry were performed using human endometrial and rat uterine tissues. 3,3-Diaminobenzidine tetrahydrochloride (DAB) immunohistochemistry [46] and dual-immunofluorescence assays [47] were based on the previously described methodology. The primary antibodies anti-AR (1:200 dilution) and anti-CYR61 (1:30 dilution) were used for DAB immunohistochemistry. The primary antibodies anti-AR (1:50 or 1:200 dilution) and Ki-67 (1:50 dilution) were used for immunofluorescence assay.

\section{Statistics}

Numerical, grouped results are presented as means \pm SEM. All data were analyzed using one-way or two-way ANOVA followed by Dunnett's post-hoc tests. For the in vivo study, two-way ANOVA was used to assess the main effects of menstrual cycle phase/endometrial hyperplasia and PCOS and to identify the interactions between them. A $p$-value less than 0.05 was considered statistically significant. Statistical analyses were performed using SPSS version 21.0 statistical software for Windows (SPSS Inc., Chicago, IL).

\section{Results}

\section{Regulation of endometrial AR expression in women regardless of PCOS condition was menstrual phase-dependent and cell type-dependent}

Western blot analysis showed that the levels of AR were lower in the secretory phase than the proliferative phase of the menstrual cycle in non-PCOS and PCOS patients and that AR protein expression was higher in PCOS patients with hyperplasia than non-PCOS patients with hyperplasia (Fig. 3A). In addition, although a tendency toward increased AR expression level did not result in a significant difference between non-PCOS and PCOS patients, the number of AR-positive cases was higher in PCOS patients in the proliferative phase $(9 / 9,100 \%)$ than non-PCOS patients in the proliferative phase $(10 / 15,66.67 \%)$. The number of AR-positive cases was similar in the secretory phase between non-PCOS $(8 / 15,53.33 \%)$ and PCOS $(1 / 2,50 \%)$ patients. Immunohistochemical 
analysis revealed weak and heterogeneous positive nuclear staining for AR in the epithelial cells in non-PCOS patients in the proliferative phase (Fig. 3B1 and B2) compared to non-PCOS patients in the secretory phase (Fig. 3D1 and D2) and to PCOS patients in all phases (Fig. 3C1, C2, E1, and E2). This nuclear distribution of AR in the epithelial cells was confirmed by immunofluorescence assay (data not shown). In line with the Western blot analysis, although AR was predominately localized in the stromal cells, an intense overall AR immunoreactivity was detected in the proliferative phase compared to the secretory phase of the menstrual cycle in non-PCOS patients (compare Fig. 3B1 and $\mathrm{B} 2$ to Fig. 3D1 and D2). In contrast, similar levels of AR immunoreactivity were seen between the proliferative (Fig. 3C1 and C2) and secretory (Fig. 3E1 and E2) phases in PCOS patients.

\section{Nuclear AR expression was increased in the epithelial cells in PCOS patients with hyper- plasia}

With endometrial hyperplasia, a significant in-

A

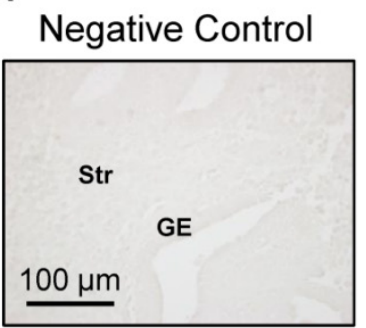

AR 1:100

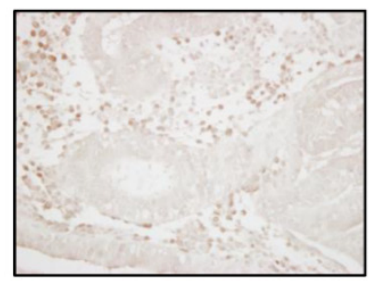

AR 1:500

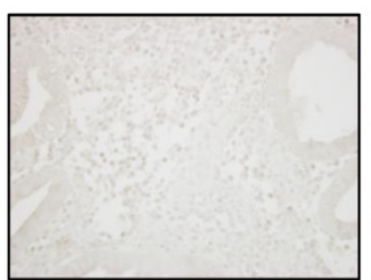

AR 1:50

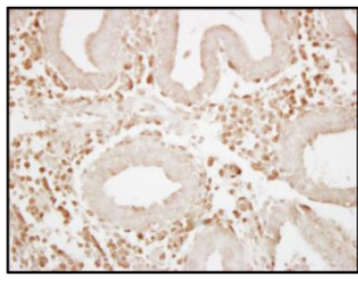

AR 1:200

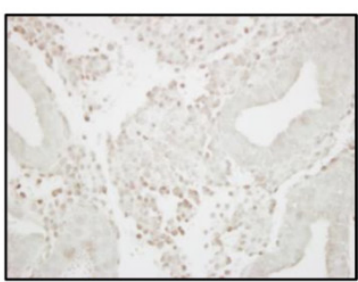

AR 1:10

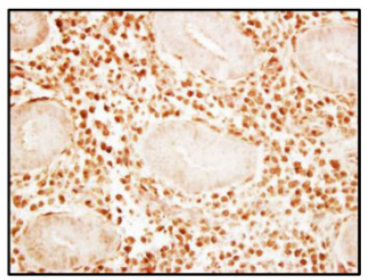

B

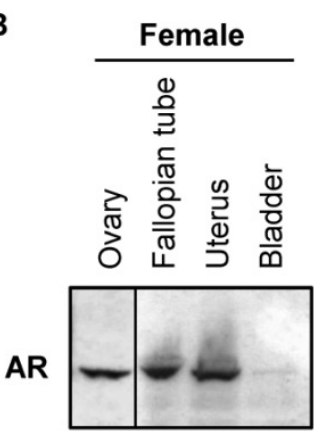

crease in AR protein level was identified in PCOS patients compared to non-PCOS patients (Fig. 3A). The number of AR-positive cases was 4/11 (36.36\%) in non-PCOS patients with hyperplasia and $3 / 3$ $(100 \%)$ in PCOS patients with hyperplasia. In contrast to non-PCOS patients with hyperplasia (Fig. 3F1 and F2), increased nuclear AR expression was detected in the epithelial cells in PCOS patients with hyperplasia although the number of epithelial AR-positive cells and the intensity of the immunoreactivity were variable (Fig. 3G1 and G2). To gain insights into the androgen-dependent in vivo regulation of nuclear expression of AR in PCOS patients with hyperplasia, an immunofluorescence assay was performed with uterine tissues from prepubescent rats treated with DHT. We found that while AR was expressed in the epithelial and stromal cells in the prepubescent rat uterus (Fig. 4A1-A4), treatment with DHT increased nuclear AR expression in the uterine cells, especially in the luminal epithelial cells (Fig. 4B1-B4). 
A

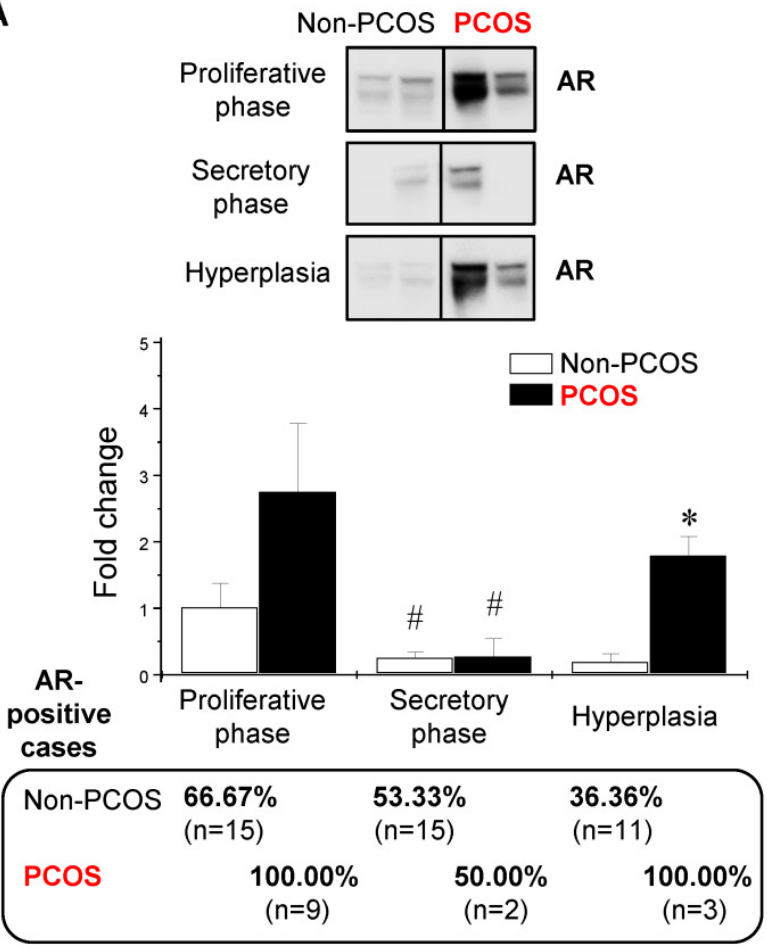

Proliferative phase
Non-PCOS
PCOS
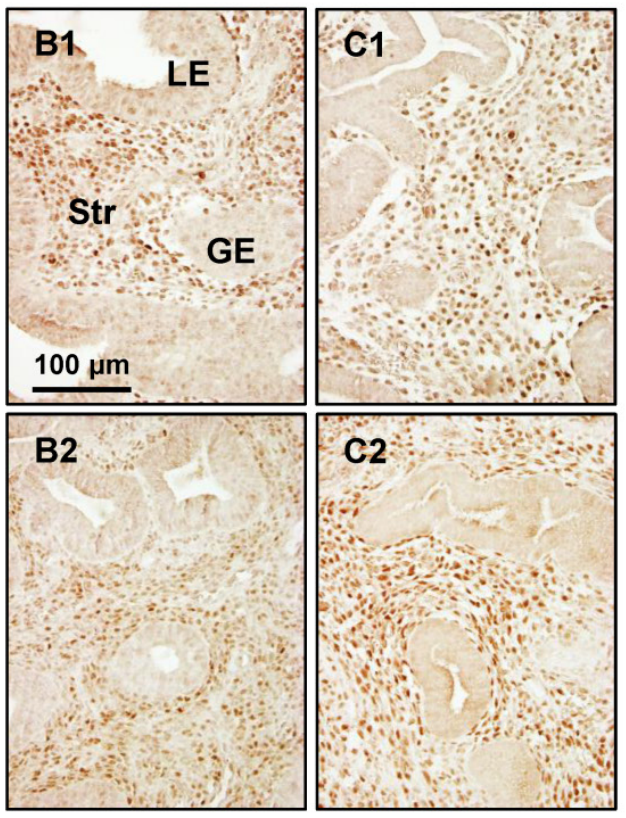
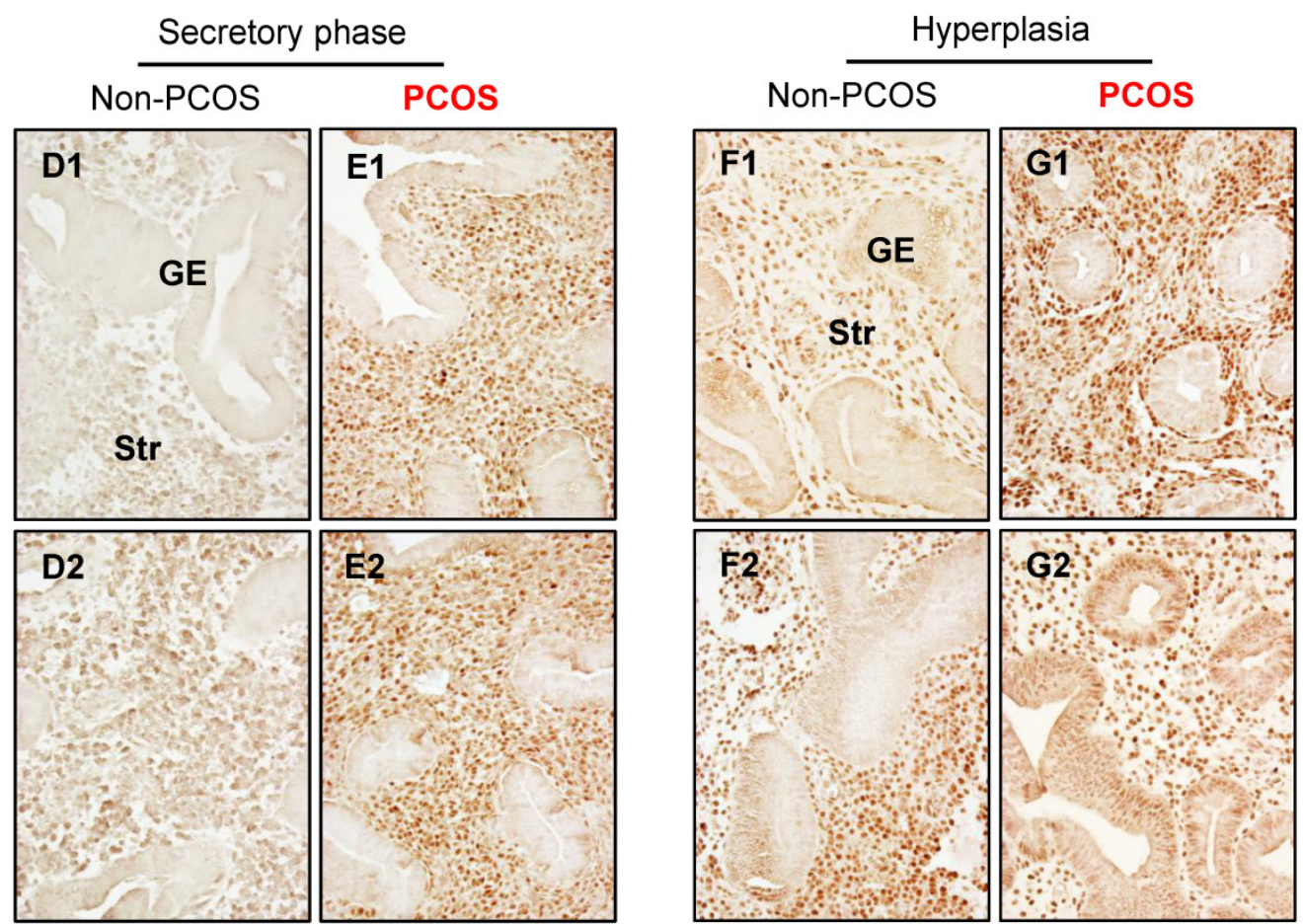

Figure 3. Differential expression and localization of endometrial AR in women with and without PCOS. Representative Western blot analysis of AR (\#5153) expression in human endometrial tissues in vivo (A). Values are expressed as mean \pm SEM. $* p<0.05$ compared to non-PCOS; \# $p<0.05$ compared to the proliferative phase. The percentages of AR-positive cases in each group are shown. Comparison of immunohistochemical staining for AR (\#5153) expression in the proliferative phase of women without PCOS (B1 and B2) and with PCOS (C1 and C2), in the secretory phase of women without PCOS (D1 and D2) and with PCOS (E1 and E2), and in women with hyperplasia without PCOS (F1 and F2) and in women with both PCOS and hyperplasia (G1 and G2). Of note, although the epithelial AR staining displays a heterogeneous pattern, nuclear expression of AR was detected in glandular epithelial cells in the proliferative phase of women without PCOS and without hyperplasia (B1), in women with hyperplasia but without PCOS (F2), and in women with both hyperplasia and PCOS (G2). The brown spots represent nuclei AR-positive glandular epithelial cells that heterogeneously coexist with AR-negative glandular epithelial cells. The numbers 1 and 2 represent images from two different patients. The findings illustrated are representative of those observed in numerous sections from multiple endometrial tissues. LE, luminal epithelial cells; GE, glandular epithelial cells; Str, stromal cells. 

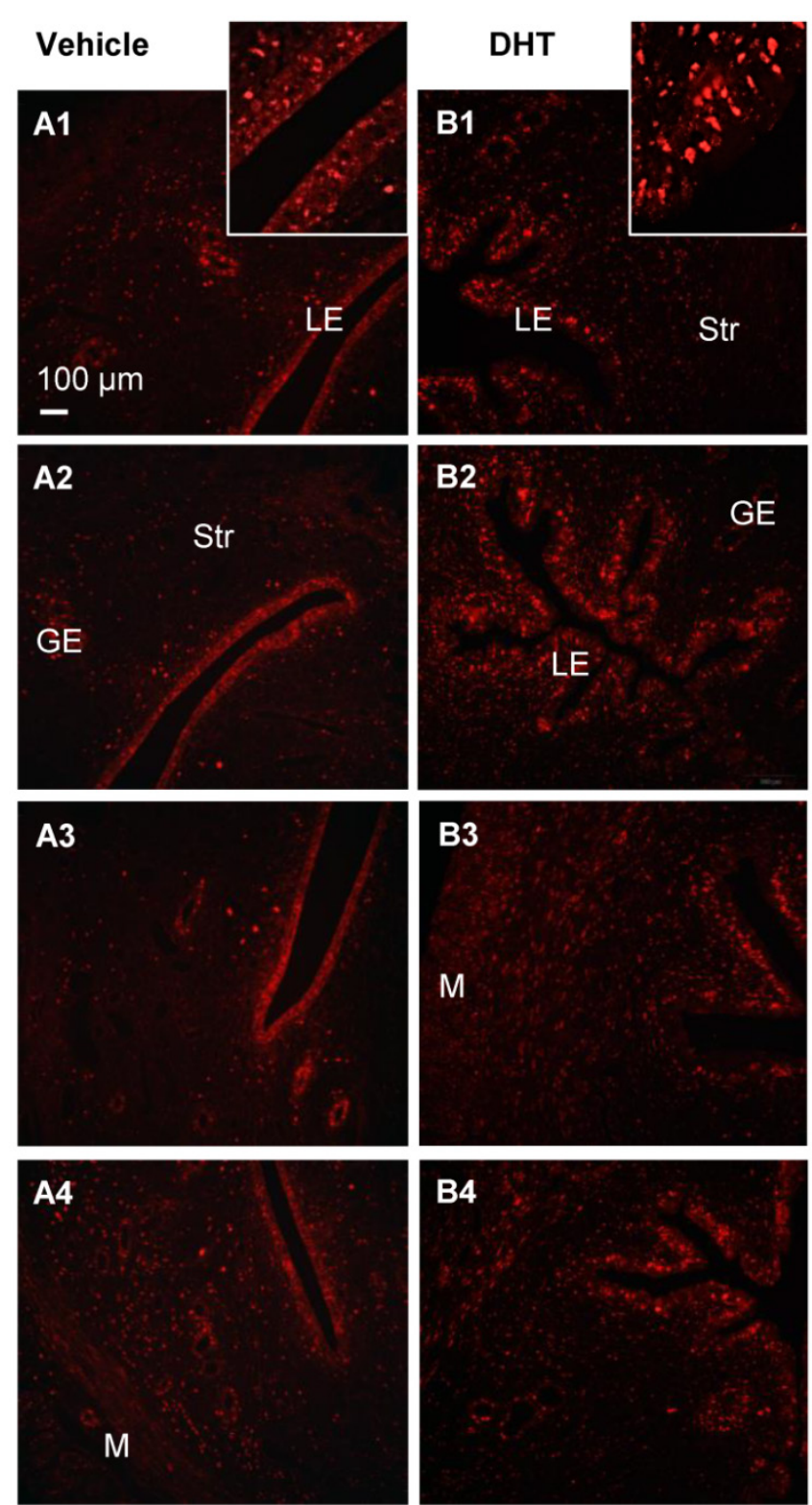

\section{B4}

Figure 4. Immunofluorescence localization of uterine AR in DHT-treated rats. Representative paraffin-embedded uterine sections in rats treated without $(A 1-4)$ and with DHT (B1-4) for one week are shown, and immunofluorescence was performed. Of note, nuclear AR (sc-816) was significantly higher in uterine epithelial cells in DHT-treated rats than those in controls. Enhanced magnifications are shown in the upper right corner of $\mathrm{A} I$ and $\mathrm{B} 1$. The findings illustrated are representative of those observed in numerous sections from multiple uterine tissues. The numbers 1-4 represents images from different rats treated with (B) and without (A) DHT. LE, luminal epithelial cells; GE, glandular epithelial cells; Str, stromal cells; M, muscle cells.

\section{p-AMPKa and AMPKa were widely expressed in both epithelial and stromal cells, but only regulation of p-AMPKa was menstrual phase-dependent and cell type-dependent}

Using the same human endometrial biopsies, Western blot analysis revealed that the levels of p-AMPKa (phosphorylated threonine 172) were higher in the secretory phase than the proliferative phase of the menstrual cycle in non-PCOS patients; however, the levels of p-AMPKa failed to change in
PCOS patients during the menstrual cycle (Fig. 5). In addition, there were no significant changes in AMPKa protein levels at different phases of the menstrual cycle in women regardless of PCOS or hyperplasia conditions (Fig. 5). We found that the ratio of p-AMPKa to AMPKa was higher in PCOS patients than non-PCOS patients in the proliferative phase. In contrast, the ratio of p-AMPKa to AMPKa was lower in PCOS patients than non-PCOS patients under hyperplasia conditions (Fig. 5).

Both p-AMPKa and AMPKa immunoreactivities were detected in the cytosol and nuclei of the endometrial cells. Although p-AMPKa (Fig. 6A1-F1) and AMPKa (Fig. 6A2-F2) were expressed in epithelial and stromal cells in women regardless of PCOS or hyperplasia conditions significantly higher levels of immunoreactivity of p-AMPKa (Fig. 6B1) and AMPKa (Fig. 6B2) were detected in the epithelial cell nuclei in PCOS patients in the proliferative phase of the menstrual cycle. There were no obvious changes to the immunoreactivity of stromal p-AMPKa (Fig. 6A1-F1) and AMPKa (Fig. 6A2-F2) expression in non-PCOS and PCOS patients. To determine the specificity of the primary antibody, immunohistochemical analysis was performed using only secondary antibodies (Fig. 6G). In line with the human results, increased expression of p-AMPKa (Fig. 7A1-B2) and AMPKa (Fig. 7C1-D2) was also observed in luminal epithelial cell nuclei in the uteri of rats treated with DHT.

\section{Activation of AR by DHT increased p2 1 cip1 expression, and suppression of $A R$ with flutamide decreased $\mathrm{p}$-AMPK $\alpha$ expression}

In order to mimic the hyperandrogenism in PCOS patients, endometrial tissues were collected from non-PCOS patients in the proliferative phase (high levels of endogenous estrogen) and treated with $100 \mathrm{nM}$ DHT. After $24 \mathrm{~h}$, the tissues were further treated with $100 \mathrm{nM}$ DHT, $100 \mathrm{nM}$ DHT combined with $10 \mu \mathrm{M}$ flutamide (an AR antagonist), or vehicle for an additional $24 \mathrm{~h}$ (Fig. 8A). p21 ${ }^{\text {Cip1 } 1}$ is considered to be an androgen-regulated response gene because its promoter contains an androgen response element [48]. Western blot analysis showed that DHT treatment increased AR and p21 ${ }^{\mathrm{Cip} 1}$ expression, and the addition of flutamide abolished the DHT-induced AR

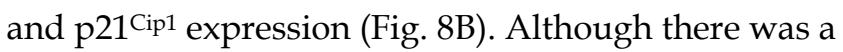
tendency for an increase in p-AMPKa expression in the endometrial tissues treated with DHT, a significant decrease in p-AMPKa expression was observed upon addition of flutamide (Fig. 8B). Neither AMPKa expression nor ratios of $\mathrm{p}$-AMPKa to AMPKa were affected by treatment with DHT and/or flutamide (Fig. 8B). 

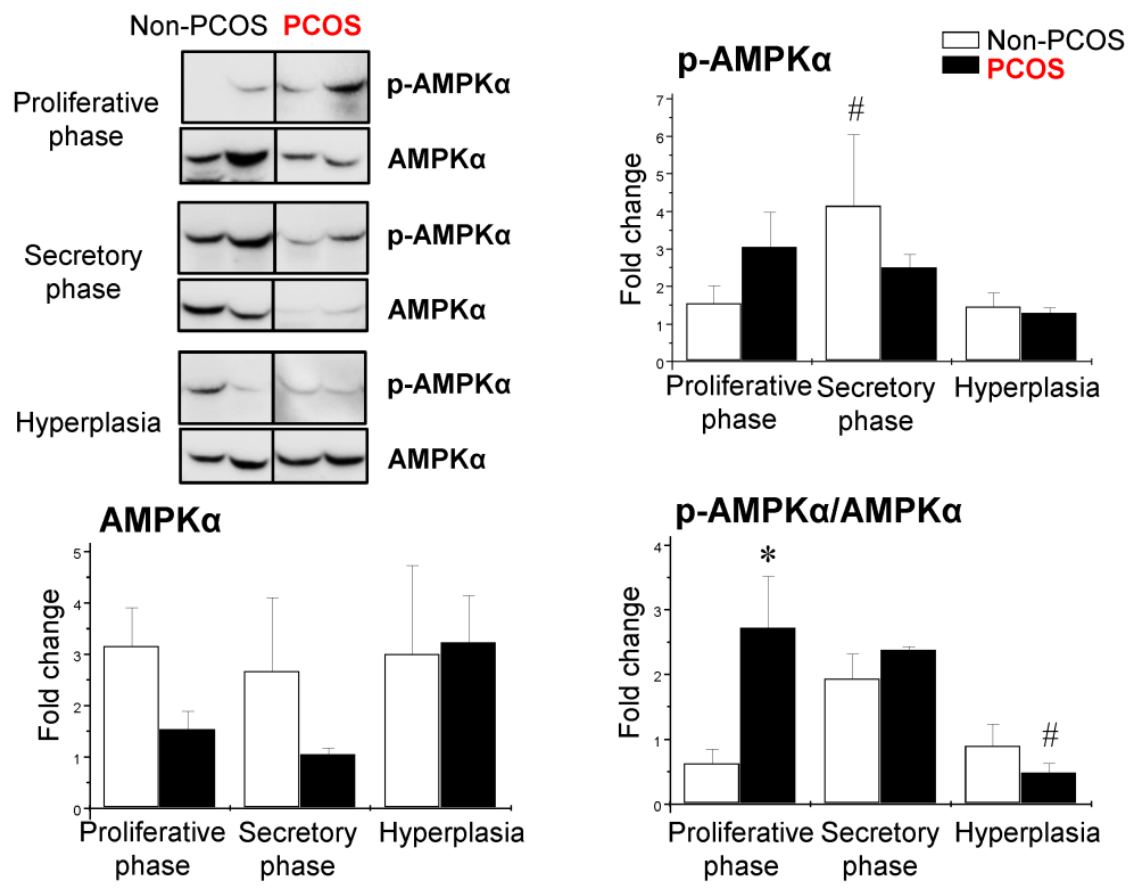

Figure 5. Differential expression of endometrial p-AMPKa and AMPKa in women with and without PCOS. Representative Western blot analysis of p-AMPK (phosphorylated threonine 172) and AMPK expression in human endometrial tissues in vivo. Values are expressed as mean \pm SEM. $* p<0.05$ compared to non-PCOS; $\#<0.05$ compared to the secretory phase.

\section{Regulation of endometrial Ki-67 expression was dependent on menstrual cycle phase and it was co-localized with AR in epithelial cells under hyperplasia conditions}

An immunofluorescence assay showed that the nuclear protein Ki-67, a cellular marker for proliferation, was present in both epithelial and stromal cells at all phases of the menstrual cycle (data not shown). The number of Ki-67-positive cells was affected by the phase of the menstrual cycle in non-PCOS and PCOS patients. Furthermore, the number of Ki-67-positive epithelial cells was lower in PCOS patients than non-PCOS patients in the secretory phase of the menstrual cycle (Fig. 9A). There was also a decreased number of Ki-67-positive epithelial cells in PCOS patients with hyperplasia compared to non-PCOS patients with hyperplasia (Fig. 9A). Under hyperplasia conditions, the numbers and immunoreactivity of AR-positive epithelial cells were higher in PCOS patients (Fig. 9C1 and C2) than non-PCOS patients (Fig. 9B1 and B2), which is in agreement with the immunochemical analysis (Fig. 3 F1-G2). In addition, a dual-immunofluorescence assay revealed that Ki-67 was co-localized with AR in epithelial cells under hyperplasia conditions in both non-PCOS (Fig. 9B1 and B2) and PCOS (Fig. 9C1 and C2) patients.
There was no co-localization between Ki-67 and AR in epithelial cells in PCOS patients without hyperplasia (Fig. 9D1 and D2). This suggests that Ki-67 expression was not dependent on androgenic regulation in the endometrial epithelial cells.

\section{Discussion}

Interactions between androgens, AR expression, and AMPK activation have been reported in other tissues and cells [49-51], but have not been investigated in human endometrium under pathophysiological conditions. This study identifies a link between the effects of androgen on endometrial AR expression and AMPKa activation in non-PCOS and PCOS patients during the menstrual cycle and/or with endometrial hyperplasia. We show that 1) endometrial AR expression levels fluctuate in non-PCOS and PCOS patients during the menstrual cycle; 2) the menstrual phase-dependent alteration of p-AMPK expression occurs in non-PCOS patients but not in PCOS patients; 3) AR expression is higher in PCOS patients than non-PCOS patients during hyperplasia while AMPKa activation (indicated by the ratio of p-AMPKa to AMPKa); and 4) the action of androgen on AMPKa activation is likely mediated through nuclear AR, especially in epithelial cells. 

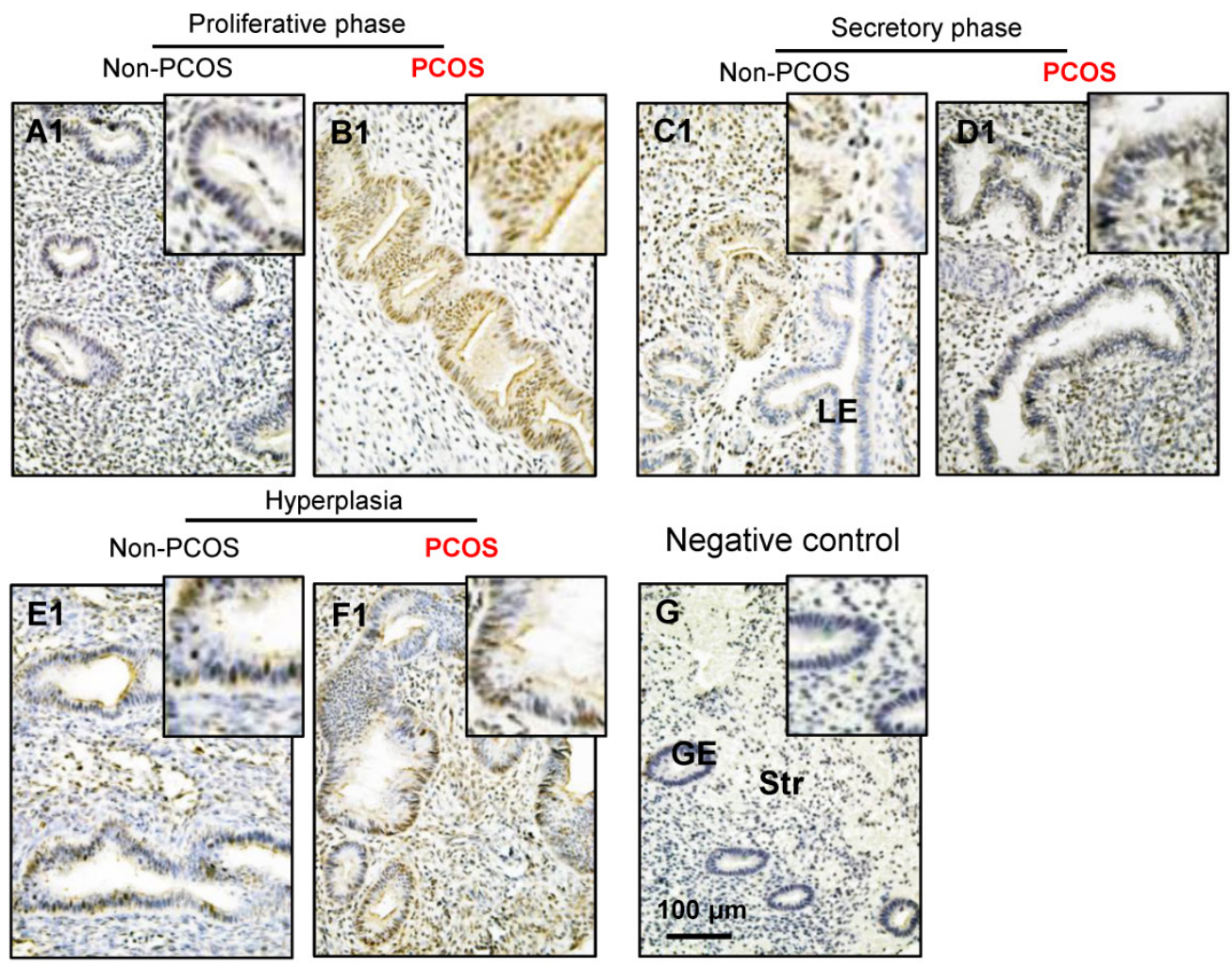

\section{Negative control}
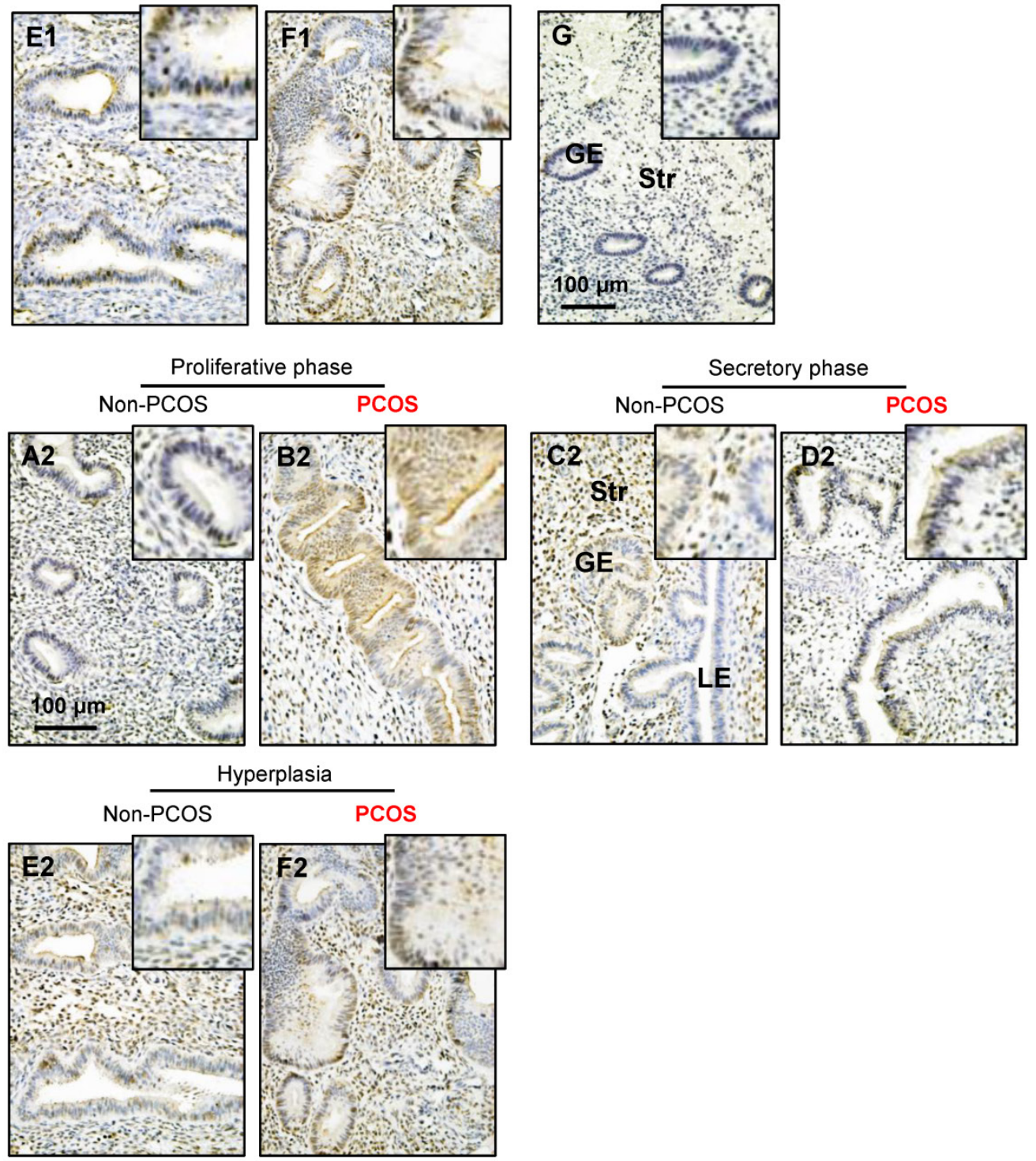

Figure 6. Distribution of p-AMPKa and AMPKa proteins in the endometrium in women with and without PCOS. Human endometrial tissues were fixed in formalin and embedded in paraffin, and immunohistochemistry was performed. Comparison of immunohistochemical staining for p-AMPK (phosphorylated threonine 172) expression (Al-FI) in the proliferative phase of women without PCOS (Al) and with PCOS (BI), in the secretory phase of women without PCOS (C1) and with PCOS (DI), in women with hyperplasia and without PCOS (EI), and in women with both hyperplasia and PCOS (FI). Of note, nuclear expression of P-AMPK was detected in glandular epithelial cells in the proliferative phase of women with PCOS $(\mathrm{Bl})$ and in the secretory phase of women without PCOS $(\mathrm{Cl})$. The same concentration of rabbit lgG instead of the primary and secondary antibodies was used as the negative control (G). Immunohistochemical staining for AMPK expression (A2-F2) is shown for the proliferative phase of women without PCOS (A2) and with PCOS (B2), the secretory stage of women without PCOS (C2) and with PCOS (D2), in women with hyperplasia and without PCOS (E2), and in women with both hyperplasia and PCOS (F2). Enhanced magnifications are shown at the upper right corners of A to G. Nuclear counterstaining with hematoxylin was performed. The images are representative of those observed in numerous sections from multiple endometrial tissues. LE, luminal epithelial cells; GE, glandular epithelial cells; Str, stromal cells. 

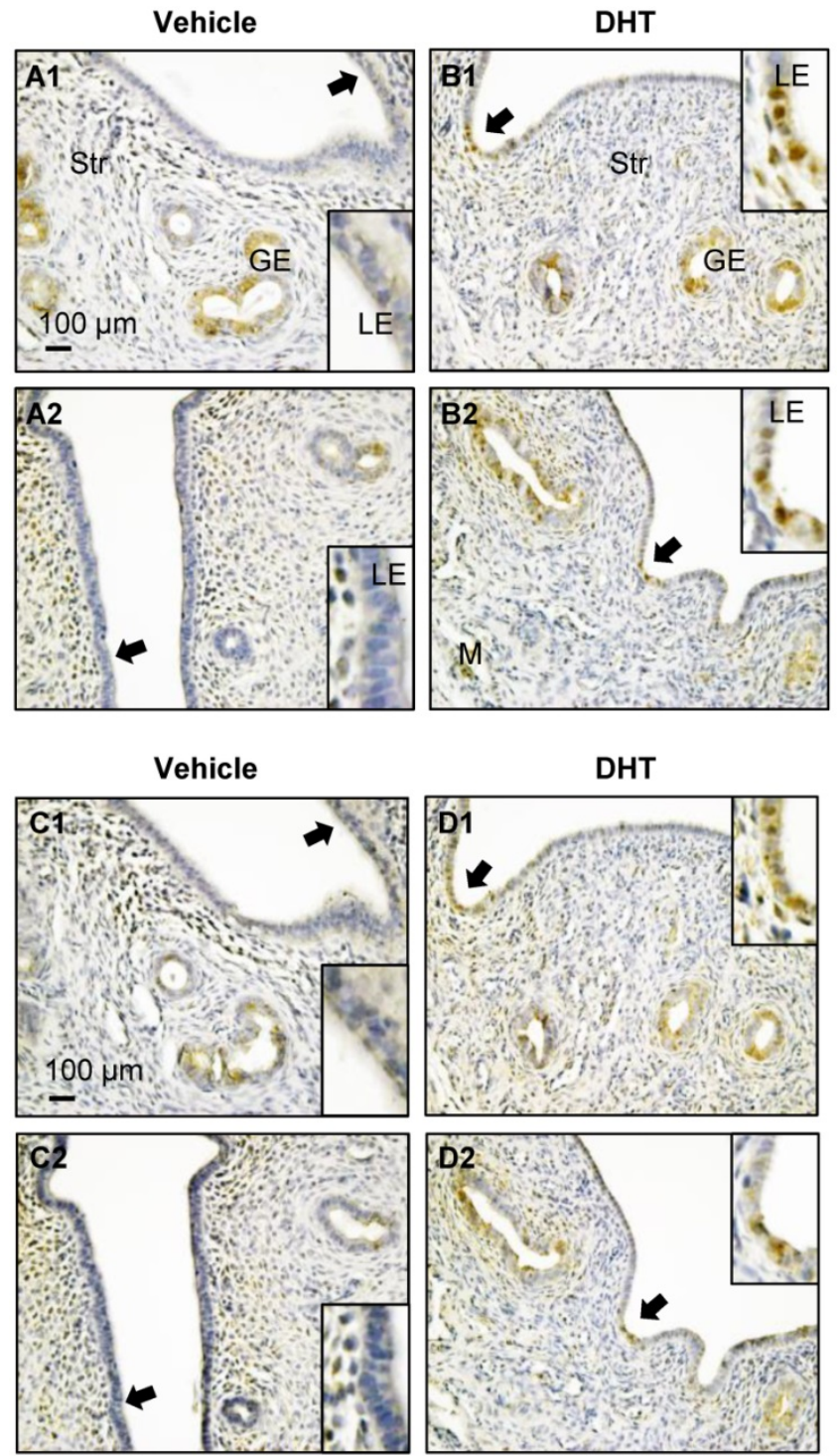

Figure 7. Distribution of uterine p-AMPK (A1-B2) and AMPK (C1-D2) proteins in DHT-treated rats. Representative paraffin-embedded uterine sections from rats treated without (A1-2 and $\mathrm{Cl}-2)$ and with $\mathrm{DHT}(\mathrm{BI}-2$ and $\mathrm{Dl}-2)$ for one week are shown, and immunohistochemistry was performed. Of note, DHT treatment resulted in increased nuclear P-AMPKa (threonine 172) and AMPKa expression in DHT-treated rats compared to controls. Enhanced magnifications are shown in the lower and upper right corners of $A$ to $D$. The images are representative of those observed in numerous sections from multiple uterine tissues. LE, luminal epithelial cells; GE, glandular epithelial cells; Str, stromal cells; M, muscle cells.

\section{Regulation of endometrial AR expression in non-PCOS and PCOS patients}

Our observation of increased AR expression in the endometrium in PCOS patients is broadly in agreement with previous reports [12, 17-21]. However, these studies did not determine whether the different phases of the menstrual cycle influence AR expression in PCOS patients. In fact, we did observe a cyclical fluctuation of AR expression in PCOS patients, although the level of AR expression was always higher in PCOS patients than non-PCOS patients. It is realized that the regulation of AR expression might vary and that different complexes of subunits might

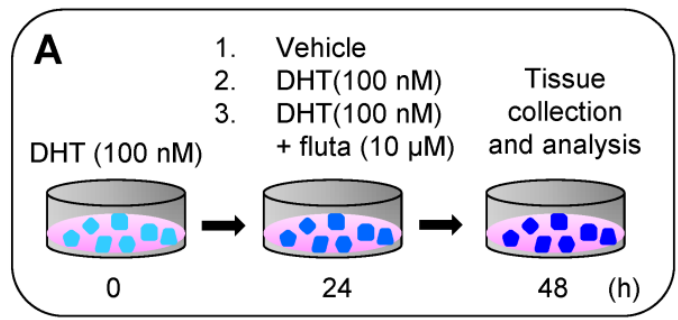

B
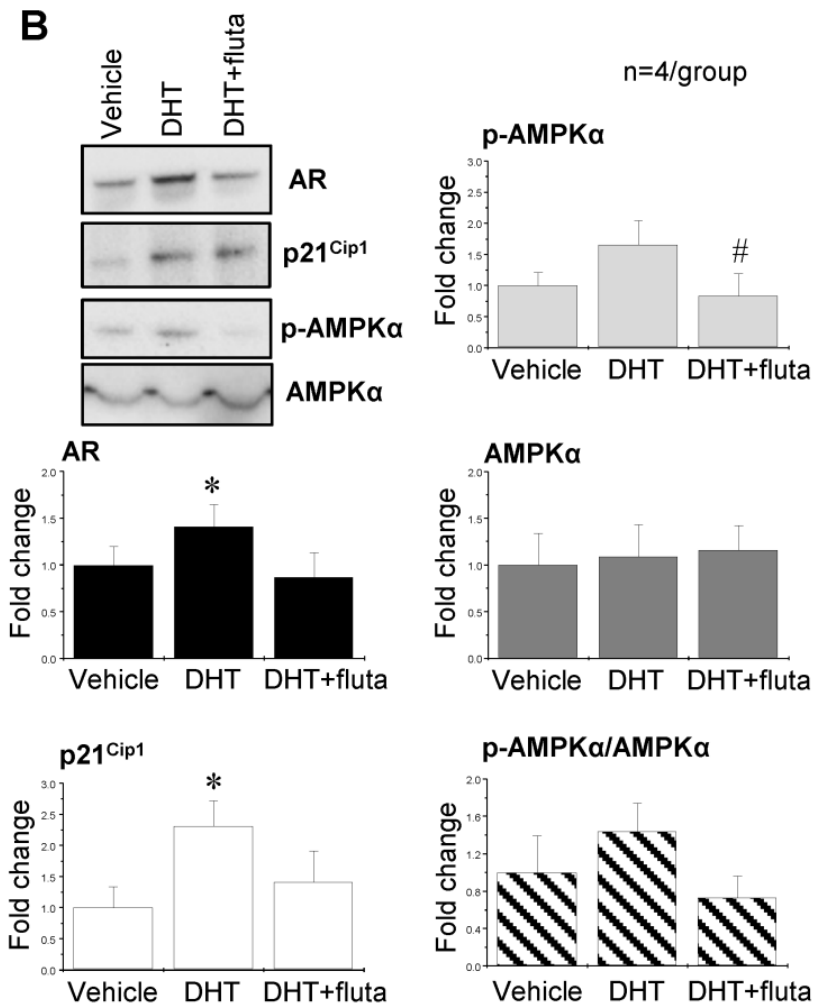

Figure 8. Effects of AR agonist and antagonist on AR, p2 1 cip1, p-AMPKa, and AMPK $\alpha$ expression in human endometrial tissues in vitro. Illustration of the in vitro experiments with $A R$ agonist and antagonist (A). Endometrial tissues from the proliferative phase of healthy women $(n=4)$ were treated with 100 $\mathrm{nM} \mathrm{DHT} \mathrm{(an} \mathrm{AR} \mathrm{agonist).} \mathrm{After} 24 \mathrm{~h}$, the culture media with DHT were removed and the endometrial explants were treated with $100 \mathrm{nM} \mathrm{DHT}$ or $100 \mathrm{nM} \mathrm{DHT}+10 \mu \mathrm{M}$ flutamide (fluta, an AR antagonist) for 24 hours. Culture media without DHT served as controls (vehicle). Representative Western blot analysis of AR, p21 Cipl, p-AMPK, and AMPK expression (B) was performed as described in the Materials and Methods. Values are expressed as mean \pm SEM. ${ }^{* *} p<0.05$ compared to vehicle; ${ }^{\#} p<0.05$ compared to DHT.

form in different tissues/cells. Common opinion holds that androgens are the primary regulators of $A R$ expression and activation [11]; however, previous studies show that the high levels of AR are associated with the proliferative phase of the menstrual cycle [12-16] and that AR expression is also regulated by estrogens in human endometrium $[52,53]$. While endogenous androgen levels [1,3] and endometrial P450 aromatase expression [54] are significantly increased in PCOS patients, it is likely that both androgen and estrogen contribute to increased endometrial AR expression in PCOS patients. However, under the estrogen-related hyperplasia condition we observed a 
significant increase in AR expression in PCOS patients compared to non-PCOS patients. Therefore, our findings indicate that androgens might play a major role in up-regulating endometrial AR protein synthesis in PCOS patients.

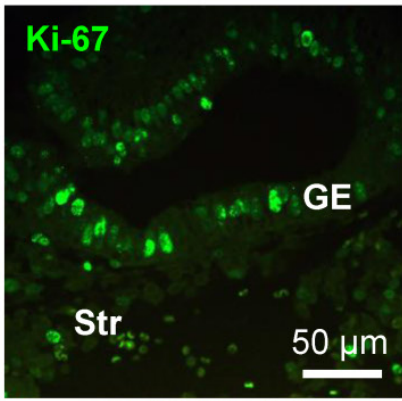

A

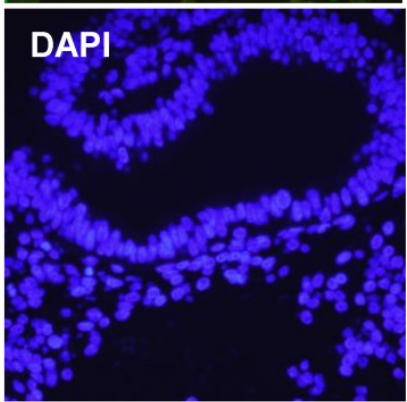

\section{Glandular epithelia}

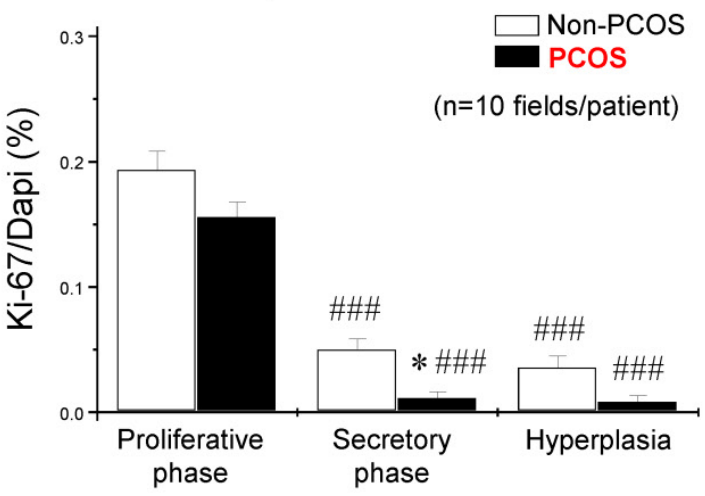

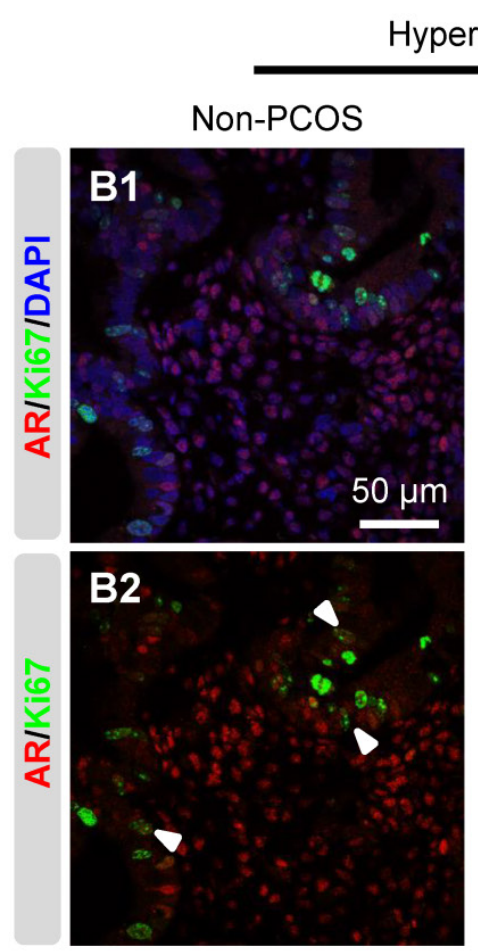

Hyperplasia
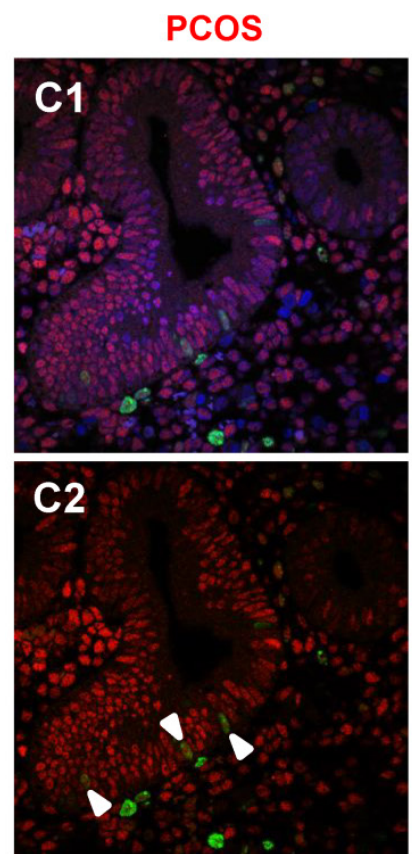

Non-hyperplasia

PCOS
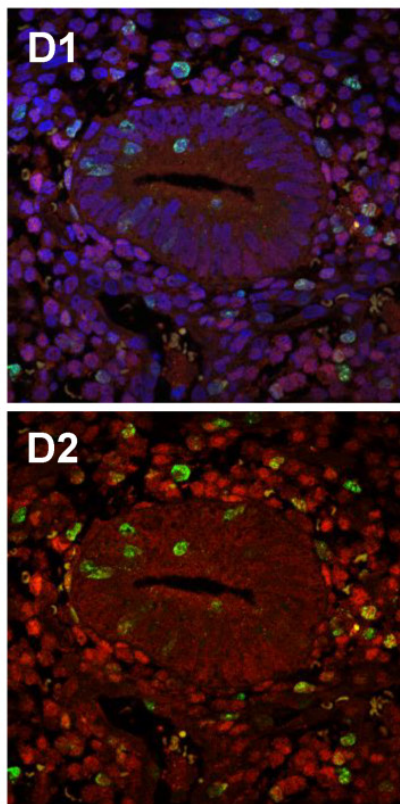

Figure 9. An association between AR and Ki-67 in endometrial epithelial cells in women with hyperplasia regardless of the presence of PCOS. Differential expression and localization of endometrial epithelial Ki-67 in women with and without PCOS (A). Values are expressed as mean \pm SEM. * $p<0.05$ compared to non-PCOS; \#\# $p<0.001$ compared to the proliferative phase. Representative immunofluorescent images of nuclear Ki-67 in endometrial epithelial and stromal cells in the proliferative phase of healthy women are shown. Localization of endometrial AR and Ki-67 in women with hyperplasia (B1 and B2) and in women with PCOS and with hyperplasia ( $C 1$ and $\mathrm{C} 2$ ) and in women with PCOS and without hyperplasia (D1 and D2). Endometrial sections were immunolabeled for AR and Ki-67 and visualized with the appropriate wavelength for AR (red) and Ki-67 (green) and then counterstained with DAPI to visualize cell nuclei (blue). Confocal microscopy revealed that AR negatively colocalized with Ki-67 in epithelial cells in women with PCOS but without hyperplasia. Arrowheads indicate colocalization of AR and Ki-67. GE, glandular epithelial cells; Str, stromal cells. 
Several molecules have been shown to display a cell specific and/or temporal expression pattern in the endometrium under physiological and pathological conditions $[9,55]$, and our localization data for AR in stromal cells in PCOS patients are consistent with previous studies [12, 20]. Endometrial AR has several proposed, but no proven, roles in human reproductive function. For instance, data from in vitro studies using the endometrial stromal cell culture suggest that the androgen-induced AR activation might participate in the anti-proliferative process against estrogen and in uterine receptivity to embryo implantation [11]. Furthermore, genetic models of loss of function of AR have demonstrated that female mice suffer from defective implantation and reduced fertility due to improper development of the uterus [56]. Therefore, it is possible that the high levels of anti-proliferative AR in the stromal cells are involved in the implantation failure and recurrent miscarriage in women with PCOS. On the other hand, DHT-induced AR expression has been shown in well-differentiated epithelial cells (Ishikawa cells) in vitro but not in isolated human endometrial stromal cells [12]. While AR is present in human endometrial epithelial cells in the proliferative phase of the menstrual cycle in vivo [[10], the present study] and in vitro [57], we observed that AR immunoreactivity was increased in particular in the epithelial cell nuclei in PCOS patients with endometrial hyperplasia. Endometrial hyperplasia is a known precursor of and marker for concurrent endometrial cancer [28], which originates in the endometrial epithelial cells [58]. It has been reported that $70 \%$ of endometrial cancer are AR positive [14], and AR immunoreactivity can be detected in PCOS patients with endometrial cancer (data not shown). Thus, we speculate that the function of epithelial AR might differ from stromal AR in the endometrium and that the activation of epithelial AR might critically affect epithelial cell transformation and endometrial cancer development.

\section{AR-mediated AMPKa activation in non-PCOS and PCOS patients}

In this study, we used Western blot and immunohistochemical analyses to evaluate the expression levels of p-AMPKa and AMPKa in the endometrium under physiological and pathological conditions. We show that the active form of AMPKa, p-AMPKa, but not AMPKa, is significantly regulated in non-PCOS patients in a menstrual phase-dependent manner. Our analysis detected no significant changes in either p-AMPKa or AMPKa expression in the endometrium in PCOS patients. This does not dispute the importance of AMPKa activation in the endometrium under disease condition. How- ever, it does reveal that the endometrial p-AMPKa/AMPKa ratio was increased in the proliferative phase of the menstrual cycle in PCOS patients compared to non-PCOS patients and it was decreased in PCOS patients with endometrial hyperplasia compared to non-PCOS patients during the secretory phase (Fig. 5).

The direct effects of androgen on AMPKa expression and activation have been described in prostate cancer cells in vitro [37]. Within the in vitro experiments of this study, we demonstrate the androgen-dependent regulation of $\mathrm{AR}$ expression and AMPKa activation in the endometrium. Unfortunately, we are unable to observe a significant effect of DHT treatment on the increased p-AMPKa expression in human endometrium in vitro, which mimics hyperandrogenism in PCOS patients in vivo. Because androgens can exert their effects through cell-membrane bound ARs [59], our findings do not rule out the possibility that AMPKa activation is dependent on acute (nongenomic) androgen stimulation in the endometrial cells. Interestingly, our data show that adding the AR antagonist flutamide along with DHT inhibits the androgenic effect of the DHT. Increased AR might function through modulation of various downstream targets such as the cell cycle regulator $\mathrm{p} 21^{\mathrm{Cip} 1}$ to influence endometrial cell proliferation and apoptosis [60].

\section{The possible role of AMPKa activation in the endometrium}

AMPK contributes to the regulation of cellular metabolism and limits cell growth [31,32]. Although a physiological implication of AMPKa activation in the endometrium is uncertain [29], we show that endometrial AMPKa activation is decreased in PCOS patients with hyperplasia compared to PCOS patients without hyperplasia. The fact that there is a similar expression pattern between p-AMPKa (the present study) and p21 ${ }^{\mathrm{Cip} 1}$ [60] in human endometrial epithelial cells during the menstrual cycle and that activation of the AMPK complex ( $\alpha \beta \gamma$ units) modulates cell cycle progression through induction of p21 ${ }^{\text {Cip1 }}$ [31] suggests the possibility that inhibition of AMPKa activation blocks cell cycle progression by suppressing p21 ${ }^{\text {Cip1 }}$ expression. Such inhibition of AMPKa would result in increased cell proliferation and hyperplasia in PCOS patients; however, further investigations are needed to test such a possibility.

In this study, we also demonstrate that p-AMPKa and AMPKa expression is primarily in the cytosolic and nuclear endometrial cells and that the immunoreactivities to these molecules are greater in the epithelial cell nuclei of PCOS patients than non-PCOS patients and in rats treated with DHT. Our 
results are in agreement with the subcellular localization data obtained by Schuler et al. [35] but are in contrast to the results obtained by Carvajal et al. [34]. The nuclear localization of p-AMPKa and AMPKa is not surprising because the antibodies against p-AMPKa and AMPKa used this study can detect both the a1 and a2 isoforms. In fact, in cells the AMPKa1 isoform appears to be dominantly expressed in the cytosol whereas the AMPKa2 isoform is largely localized in the nuclei [33]. Importantly, studies of mutant mice lacking various AMPKa isoforms suggest that AMPKa2, but not AMPKa1, is essential for energy metabolism and insulin sensitivity [36]. Future studies are needed to clarify the functional differences between the two AMPKa isoforms in the endometrium.

\section{Conclusion}

Clinical evidence indicates that high expression of AR might contribute to PCOS pathogenesis [61]. In order to develop effective treatments for the endometrial dysfunction seen in women with PCOS, it is important to understand exactly how androgen-AR signalling leads to different molecular changes in the endometrium and to endometrium-related reproductive outcomes in these patients. In conclusion, our data add novel descriptive insights into AR expression and AMPKa activation in women under physiological and pathological conditions. We report for the first time that AR expression and AMPKa activation depend on menstrual cycle phase and the presence of PCOS, and AR-mediated regulation of AMPKa activation might play a role in the development of endometrial hyperplasia in women with PCOS.

\section{Acknowledgments}

This work was supported by the Swedish Medical Research Council (5859 and 10380), the Swedish federal government under the LUA/ALF agreement (ALFGBG-147791), Jane and Dan Olsson's Foundation, the Hjalmar Svensson Foundation, the Åke-Wiberg Foundation, and Clas Groschinsky's Foundation to HB and RS, as well as the Shanghai Committee of Science and Technology, China (15411964700), and the Scientific Research Project of Shanghai Municipal Health Bureau, China (20134264), to XL. The authors thank the Centre for Cellular Imaging of the Sahlgrenska Academy at Gothenburg University, Sweden.

\section{Author contribution}

XL, BP, PC, MH, HPY, YRG, HYJ, and RS participated in tissue collection, funded the experiments, and analyzed the data. XL, BP, PC and RS prepared the figures and participated in the discussion. RS was responsible for the conception and design of the research, interpreting the data, and drafting, editing and revising the manuscript. YF and $\mathrm{HB}$ contributed to interpretation of the data, and participated in the discussion. All authors approved the final submitted version of the manuscript.

\section{Competing Interests}

The authors indicate no potential conflicts of interest.

\section{References}

[1] Norman RJ, Dewailly D, Legro RS, et al. Polycystic ovary syndrome. Lancet. 2007; 370: 685-697.

[2] Moran LJ, Hutchison SK, Norman RJ, et al. Lifestyle changes in women with polycystic ovary syndrome. Cochrane Database Syst Rev. 2011; CD007506.

[3] Ehrmann DA. Polycystic ovary syndrome. N Engl J Med. 2005; 352: 1223-36.

[4] Li X, Feng Y, Lin JF, et al. Endometrial progesterone resistance and PCOS. J Biomed Sci. 2014; 21: 2

[5] Homburg R. Management of infertility and prevention of ovarian hyperstimulation in women with polycystic ovary syndrome. Best Pract Res Clin Obstet Gynaecol 2004; 18: 773-788.

[6] Chakraborty P, Goswami SK, Rajani S, et al. Recurrent pregnancy loss in polycystic ovary syndrome: role of hyperhomocysteinemia and insulin resistance. PLoS One. 2013; 8: e64446.

[7] Shang K, Jia X, Qiao J, et al. Endometrial abnormality in women with polycystic ovary syndrome. Reprod Sci. 2012; 19: 674-83.

[8] Li X, Guo JR, Lin JF, et al. Combination of Diane-35 and metformin to treat early endometrial carcinoma in PCOS women with insulin resistance. J Cancer. 2014; 5: 173-81.

[9] Kim JJ, Kurita T and Bulun SE. Progesterone action in endometrial cancer, endometriosis, uterine fibroids, and breast cancer. Endocr Rev 2013; 34: 130-62.

[10] Critchley HO and Saunders PT. Hormone receptor dynamics in a receptive human endometrium. Reprod Sci. 2009; 16: 191-9.

[11] Cloke B and Christian M. The role of androgens and the androgen receptor in cycling endometrium. Mol Cell Endocrinol. 2012; 358: 166-75.

[12] Apparao KB, Lovely LP, Gui Y, et al. Elevated endometrial androgen receptor expression in women with polycystic ovarian syndrome. Biol Reprod. 2002; 66: 297-304.

[13] Horie K, Takakura K, Imai K, et al. Immunohistochemical localization of androgen receptor in the human endometrium, decidua, placenta and pathological conditions of the endometrium. Hum Reprod. 1992; 7: 1461-6.

[14] Ito K, Suzuki T, Akahira J, et al. Expression of androgen receptor and 5alpha-reductases in the human normal endometrium and its disorders. Int J Cancer. 2002; 99: 652-7.

[15] Mertens HJ, Heineman MJ, Koudstaal J, et al. Androgen receptor content in human endometrium. Eur J Obstet Gynecol Reprod Biol. 1996; 70: 11-3.

[16] Brenner RM, Slayden OD, Nayak NR, et al. A role for the androgen receptor in the endometrial antiproliferative effects of progesterone antagonists. Steroids. 2003; 68: 1033-9.

[17] Maliqueo M, Clementi M, Gabler F, et al. Expression of steroid receptors and proteins related to apoptosis in endometria of women with polycystic ovary syndrome. Fertil Steril. 2003; 80 Suppl 2: 812-9.

[18] Quezada S, Avellaira C, Johnson MC, et al. Evaluation of steroid receptors, coregulators, and molecules associated with uterine receptivity in secretory endometria from untreated women with polycystic ovary syndrome. Fertil Steril. 2006; 85: 1017-26.

[19] Villavicencio A, Bacallao K, Avellaira C, et al. Androgen and estrogen receptors and co-regulators levels in endometria from patients with polycystic ovarian syndrome with and without endometrial hyperplasia. Gynecol Oncol. 2006; 103: 307-14.

[20] Gonzalez D, Thackeray H, Lewis PD, et al. Loss of WT1 expression in the endometrium of infertile PCOS patients: a hyperandrogenic effect? J Clin Endocrinol Metab. 2012; 97: 957-66.

[21] Li X, Cui P, Jiang HY, et al. Reversing the reduced level of endometrial GLUT4 expression in polycystic ovary syndrome: a mechanistic study of metformin action. Am J Transl Res. 2015; 7: 574-86.

[22] Gambineri A, Patton L, Vaccina A, et al. Treatment with flutamide, metformin, and their combination added to a hypocaloric diet in overweight-obese women with polycystic ovary syndrome: a randomized, 12-month, placebo-controlled study. J Clin Endocrinol Metab. 2006; 91: 3970-80.

[23] Gambineri A, Pelusi C, Genghini S, et al. Effect of flutamide and metformin administered alone or in combination in dieting obese women with polycystic ovary syndrome. Clin Endocrinol (Oxf). 2004; 60: 241-9.

[24] Ibanez L and de Zegher F. Low-dose combination of flutamide, metformin and an oral contraceptive for non-obese, young women with polycystic ovary syndrome. Hum Reprod. 2003; 18: 57-60. 
[25] Ibanez L and de Zegher F. Low-dose flutamide-metformin therapy for hyperinsulinemic hyperandrogenism in nonobese adolescents and women. Fertil Steril. 2006; 86 Suppl 1: S24-5.

[26] Ibanez L and de Zegher F. Low-dose flutamide-metformin therapy for hyperinsulinemic hyperandrogenism in non-obese adolescents and women. Hum Reprod Update. 2006; 12: 243-52.

[27] Sahin I, Serter R, Karakurt F, et al. Metformin versus flutamide in the treatment of metabolic consequences of non-obese young women with polycystic ovary syndrome: a randomized prospective study. Gynecol Endocrinol. 2004; 19: 115-24.

[28] Armstrong AJ, Hurd WW, Elguero S, et al. Diagnosis and management of endometrial hyperplasia. J Minim Invasive Gynecol. 2012; 19: 562-71.

[29] Shao R, Li X, Feng Y, et al. Direct effects of metformin in the endometrium: a hypothetical mechanism for the treatment of women with PCOS and endometrial carcinoma. J Exp Clin Cancer Res. 2014; 33: 41.

[30] Grahame Hardie D. AMP-activated protein kinase: a key regulator of energy balance with many roles in human disease. J Intern Med. 2014; 276: 543-59.

[31] Sanli T, Steinberg GR, Singh G, et al. AMP-activated protein kinase (AMPK) beyond metabolism: a novel genomic stress sensor participating in the DNA damage response pathway. Cancer Biol Ther. 2014; 15: 156-69.

[32] Hardie DG, Ross FA and Hawley SA. AMPK: a nutrient and energy sensor that maintains energy homeostasis. Nat Rev Mol Cell Biol. 2012; 13: 251-62.

[33] Shirwany NA and Zou MH. AMPK: a cellular metabolic and redox sensor. A minireview. Front Biosci. 2014; 19: 447-74.

[34] Carvajal R, Rosas C, Kohan K, et al. Metformin augments the levels of molecules that regulate the expression of the insulin-dependent glucose transporter GLUT4 in the endometria of hyperinsulinemic PCOS patients. Hum Reprod. 2013; 28: 2235-44.

[35] Schuler KM, Rambally BS, DiFurio MJ, et al. Antiproliferative and metabolic effects of metformin in a preoperative window clinical trial for endometrial cancer. Cancer Med. 2015; 4: 161-73.

[36] Long YC and Zierath JR. AMP-activated protein kinase signaling in metabolic regulation. J Clin Invest. 2006; 116: 1776-83.

[37] Brown KA, Samarajeewa NU and Simpson ER. Endocrine-related cancers and the role of AMPK. Mol Cell Endocrinol. 2013; 366: 170-9.

[38] Noyes RW, Hertig AT and Rock J. Dating the endometrial biopsy. Am J Obstet Gynecol. 1975; 122: 262-3.

[39] Cui P, Li X, Wang X, et al. Lack of cyclical fluctuations of endometrial GLUT4 expression in women with polycystic ovary syndrome: Evidence for direct regulation of GLUT4 by steroid hormones. BBA Clin. 2015; 4: 85-91.

[40] Rotterdam ESHRE/ASRM-Sponsored PCOS consensus workshop group. Revised 2003 consensus on diagnostic criteria and long-term health risks related to polycystic ovary syndrome (PCOS). Hum Reprod. 2004; 19: 41-7.

[41] Marshall E, Lowrey J, MacPherson S, et al. In silico analysis identifies a novel role for androgens in the regulation of human endometrial apoptosis. J Clin Endocrinol Metab. 2011; 96: E1746-55.

[42] Wen J, Zhu H, Murakami S, et al. Regulation of A Disintegrin And Metalloproteinase with ThromboSpondin repeats-1 expression in human endometrial stromal cells by gonadal steroids involves progestins, androgens, and estrogens. J Clin Endocrinol Metab. 2006; 91: 4825-35.

[43] Feng Y, Weijdegard B, Wang T, et al. Spatiotemporal expression of androgen receptors in the female rat brain during the oestrous cycle and the impact of exogenous androgen administration: a comparison with gonadally intact males. Mol Cell Endocrinol. 2010; 321: 161-74.

[44] Feng Y, Johansson J, Shao R, et al. Hypothalamic neuroendocrine functions in rats with dihydrotestosterone-induced polycystic ovary syndrome: effects of low-frequency electro-acupuncture. PLoS One. 2009; 4: e6638.

[45] Shao R, Norstrom A, Weijdegard B, et al. Distinct Expression Pattern of Dicer1 Correlates with Ovarian-Derived Steroid Hormone Receptor Expression in Human Fallopian Tubes during Ovulation and the Midsecretory Phase. J Clin Endocrinol Metab. 2011; 96: E869-77.

[46] Shao R, Ljungstrom K, Weijdegard B, et al. Estrogen-induced upregulation of $\mathrm{AR}$ expression and enhancement of AR nuclear translocation in mouse fallopian tubes in vivo. Am J Physiol Endocrinol Metab. 2007; 292: E604-14.

[47] Shao R, Weijdegard B, Fernandez-Rodriguez I, et al. Ciliated epithelial-specific and regional-specific expression and regulation of the estrogen receptor-beta2 in the fallopian tubes of immature rats: a possible mechanism for estrogen-mediated transport process in vivo. Am J Physiol Endocrinol Metab. 2007; 293: E147-58

[48] Lu S, Liu M, Epner DE, et al. Androgen regulation of the cyclin-dependent kinase inhibitor p21 gene through an androgen response element in the proximal promoter. Mol Endocrinol. 1999; 13: 376-84.

[49] Tennakoon JB, Shi Y, Han JJ, et al. Androgens regulate prostate cancer cell growth via an AMPK-PGC-1alpha-mediated metabolic switch. Oncogene. 2014; 33: 5251-61.

[50] Shen M, Zhang Z, Ratnam M. et al. The interplay of AMP-activated protein kinase and androgen receptor in prostate cancer cells. J Cell Physiol. 2014; 229: 688-95.

[51] McInnes KJ, Corbould A, Simpson ER, et al. Regulation of adenosine $5^{\prime}$,monophosphate-activated protein kinase and lipogenesis by androgens contributes to visceral obesity in an estrogen-deficient state. Endocrinology. 2006; 147: 5907-13.

[52] Fujimoto J, Nishigaki M, Hori M, et al. Effects of Estradiol and Testosterone on the Synthesis, Expression and Degradation of Androgen Receptor in Human Uterine Endometrial Fibroblasts. J Biomed Sci. 1995; 2: 160-5.
[53] Fujimoto J, Nishigaki M, Hori M, et al. Biological implications of estrogen and androgen effects on androgen receptor and its mRNA levels in human uterine endometrium. Gynecol Endocrinol. 1995; 9: 149-55.

[54] Zhao PL, Zhang QF, Yan LY, et al. Functional investigation on aromatase in endometrial hyperplasia in polycystic ovary syndrome cases. Asian Pac J Cancer Prev. 2014; 15: 8975-9.

[55] Amant F, Moerman P, Neven P, et al. Endometrial cancer. Lancet 2005; 366: 491-505.

[56] Chang C, Lee SO, Wang RS, Yeh S and Chang TM. Androgen receptor (AR) physiological roles in male and female reproductive systems: lessons learned from AR-knockout mice lacking AR in selective cells. Biol Reprod. 2013; 89: 21.

[57] Tuckerman EM, Okon MA, Li T, et al. Do androgens have a direct effect on endometrial function? An in vitro study. Fertil Steril. 2000; 74: 771-9.

[58] Horn LC, Meinel A, Handzel R, et al. Histopathology of endometrial hyperplasia and endometrial carcinoma: an update. Ann Diagn Pathol. 2007; 11: 297-311.

[59] Lang F, Alevizopoulos K and Stournaras C. Targeting membrane androgen receptors in tumors. Expert Opin Ther Targets. 2013; 17: 951-63.

[60] Toki T, Mori A, Shimizu M, et al. Localization of apoptotic cells within the human endometrium and correlation between apoptosis and p21 expression. Mol Hum Reprod. 1998; 4: 1157-64.

[61] Walters KA. Role of androgens in normal and pathological ovarian function. Reproduction. 2015; 149: R193-218. 